\title{
NanoCharacterization of Double PN Heterojunctions in Photoelectrochemical Devices
}

Dalaver Anjum ${ }^{1}$ and Purushothaman Varadhan ${ }^{2}$

${ }^{1}$ Khalifa University, Abu Dhabi, Abu Dhabi, United Arab Emirates, ${ }^{2}$ King Abdullah University of Science \& Technology (KAUST), Thuwal, Makkah, Saudi Arabia

The use of fossil fuel energy resources has left has impacted the ecosystem of earth in many ways. In spite of this, the world will run out of it in the matter of few decades if continue to be used at its pace [1]. Therefore, a great amount of efforts is spent for developing alternative energy resources. In this regard, hydrogen $\left(\mathrm{H}_{2}\right)$ is an excellent energy carrier and therefore delivers energy in a very efficient manner. Therefore, it has a great potential to play a key role in next generation energy resources. Hydrogen can be produced way by splitting water with solar light under the process of photoelectrochemical (PEC) [2]. A number of challenges exist to PEC technology including the life-time of hydrogen-producing systems, efficiency, and high cost. It has been reported recently that III-V double-heterojunction semiconductor based hydrogen-producing PEC systems are demonstrated to have improved life-time and efficiency as compared to their earlier counterparts [3]. The dramatic improvements in the systems are made by employing an innovative way of decoupling the interfaces of optical absorbing materials with electrocatalytic parts of PEC systems. The nanoscale characterization of double heterojunctions in the working devices is vital to the performance of PEC systems and should be carried out using an elaborate technique such as transmission electron microscopy (TEM). In this report, TEM-analysis of a working gallium arsenide (GaAs) and indium gallium phosphide ( $\mathrm{InGaP}$ ) based double heterojunction PEC system. TEM specimens of the PEC devices were prepared using focused ion beam (FIB) scanning electron microscope (SEM) of model Helios $\mathrm{G}^{4}$ from Thermo-Fisher Scientific. The analysis of the prepared specimens was then carried out using aberration-corrected TEM of model Titan 80-300 ST equipped with energy-filter of model GIF Quantum 966. Moreover, the analysis was carried out by setting the accelerating voltage to $300 \mathrm{kV}$ aberration corrected scanning TEM (AC-STEM) mode of the microscope.

In this report, we present a TEM NanoCharacterization study of $\mathrm{p} / \mathrm{n}$ junctions in the optimized III-V based PEC devices [4]. FIB prepared TEM specimens of devices were first analyzed using AC-STEM and the presented results are Figure 1 (a). Both GaAs an InGaP based p/n junctions are labeled therein. The quality of epitaxial layers present in the $\mathrm{p} / \mathrm{n}$ heterojunctions was investigated by acquiring higher magnification images. In this regard, Figure 1 (b) contains a InGaP p/n junction image acquired from dotted black-color box shown in Figure 1 (a). Similarly, Figure 1 (c) contains an image acquired from GaAs p/n junction. The combined results corroborate a nice epitaxial growth of layers. Moreover, the corresponding EDS analysis (not presented herein) of layers confirm the sequence of types of elements in each heterojunction. Strain determination in the epitaxial layers is always of great importance to the device performance and hence was also investigated in this study. It was completed by applying geometrical phase analysis (GPA) to aberration corrected high-resolution STEM (HRSTEM) images of both $\mathrm{p} / \mathrm{n}$ heterojunctions in PEC devices and the results are presented in Figure 2. Figure 2 (a) and Figure 2 (c) contain the HRSTEM and strain map in the growth for the InGaP p/n heterojunction, respectively. It can be noticed that most the layer contained negligible amount of strain in the growth direction except one layer i.e. AlGaAs and found to be about $6 \%$ compressively strained as compared to the contacting layers. In the way, the Figure 2 (b) and Figure 2 (d) contain HRSTEM and strain in the growth direction for GaAs p/n junction, respectively. Strain maps of GaAs p/n junction did not show the presence of tensile or compressive strained layers. 
In summary, the combined FIB-SEM and TEM analysis allow investigating the synthesized PEC devices at nanoscale. The analysis enables to determine the quality of epitaxy, elemental composition, and strain mapping of epitaxial layers. In conclusion, the presented results imply the paramount importance TEM NanoCharacterization to development of next generation of PEC devices.

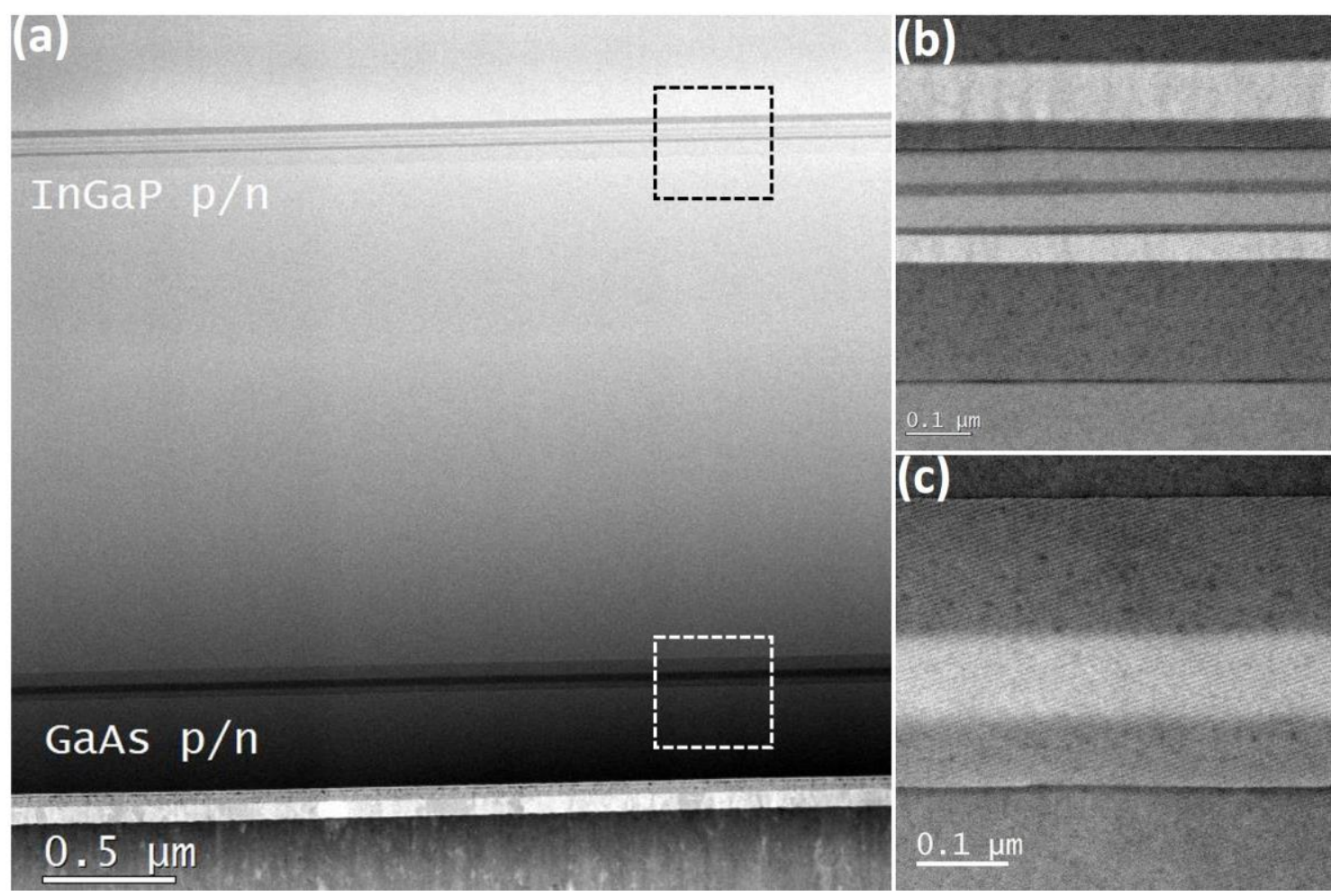

Figure 1. AC-STEM analysis of PEC device. (a) Size of PEC device and the location of $\mathrm{p} / \mathrm{n}$ heterojunctions in the synthesized devices. (b) STEM image of InGaP based $\mathrm{p} / \mathrm{n}$ heterojunction. (c) STEM image of GaAs based $\mathrm{p} / \mathrm{n}$ heterojunction. 


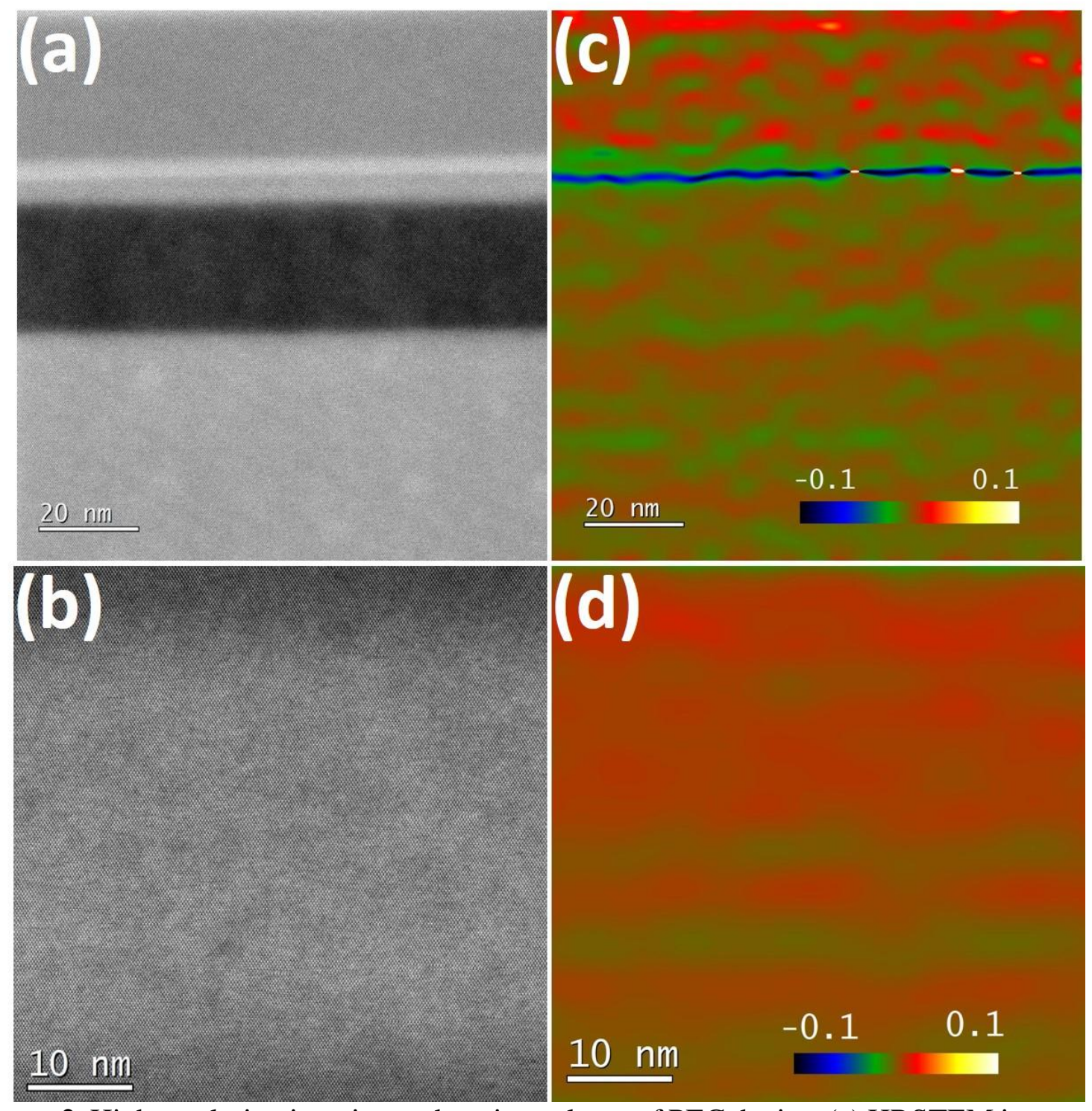

Figure 2. High resolution imaging and strain analyses of PEC device. (a) HRSTEM image of InGaP based p/n heterojunction. (b) The corresponding strain map generated by applying GPA on HRSTEM image of InGaP $\mathrm{p} / \mathrm{n}$ junction in Figure 2 (a). (d) The corresponding strain map generated by applying GPA on HRSTEM image of GaAs p/n junction in Figure 2 (b).

\section{References}

[1] Robert L. Bradley Jr., Are We Running Out of Oil?, Property and Environment Research Center, (2004), pp1-6.

[2] Fatwa F. Abdi et al., Efficient solar water splitting by enhanced charge separation in a bismuth vanadate-silicon tandem photoelectrode. Nature Communications 4, 2195 (2013).

[3] Shu $\mathrm{Hu}$ et al., Amorphous $\mathrm{TiO} 2$ coatings stabilize $\mathrm{Si}$, GaAs, and $\mathrm{GaP}$ photoanodes for efficient water oxidation, Science 344, (2014), pp1005-1009.

[4] Purushothaman Varadhan et al., An efficient and stable photoelectrochemical system with 9\% solarto-hydrogen conversion efficiency via InGaP/GaAs double junction, Nature Communications 10 (1), (2019). 
https://doi.org/10.1017/S1431927620018000 Published online by Cambridge University Press 